\title{
Pulp property development Part I: Interlacing under- sampled pulp properties and TMP process data using piece-wise linear functions
}

Anders Karlström, Jan Hill, Rita Ferritsius, and Olof Ferritsius

KEYWORDS: Pulp quality models, TMP refiners,

Piece-wise linear functions

SUMMARY: Thermo-Mechanical Pulp (TMP) refiners served in this work as an example of how to combine oversampled and undersampled variables on a common timeframe for further analysis.

To get more information from the refining process, temperature measurement arrays in the refining zones were studied alongside process measurements such as motor load, production rates, plate gaps, dilution waters, pulp properties and manually measured blow-line consistency.

The undersampled data set consisted of 63 laboratory samples obtained at a Swedish TMP mill, which were tested for tensile index, mean fiber length and Somerville shives content. The pulp samples were obtained at five different periods during three months to cover a large dynamic operating window.

The data set was expanded using a piece-wise linear approach. The measurements inside the refining zone were shown to be important variables when interlacing the undersampled pulp properties with the oversampled process data set, consisting of 350000 samples.

Use of an extended entropy model provided a palette of information about the process conditions inside the refining zone. Particularly, the residence time and the consistency in the refining zones were essential for the pulp property development, as a link between the refining segment pattern used and the current state of refiner operation.

\section{ADDRESSES OF THE AUTHORS:}

Anders Karlström ${ }^{1}$ (anderska@chalmers.se ), Sweden Jan Hill² (jan.hill@qtab.se ), Sweden

Rita Ferritsius ${ }^{3}$ (rita.ferritsius@storaenso.com), Sweden Olof Ferritsius $^{3}$ (olof.ferritsius@miun.se), Sweden

${ }^{1}$ Chalmers University of Technology, Göteborg, Sweden, ${ }^{2}$ QualTech AB, Tyringe, ${ }^{3}$ Mid Sweden University, Sweden

\section{Corresponding author: Anders Karlström}

During the latest decade, the trend in pulp and paper has been to connect traditional measurement devices and distributed control applications with mill-wide systems to provide databases for deeper process analysis. Such applications produce a data deluge that severely stresses the available analog-to-digital converters and communication bandwidth. As a consequence, important theoretical efforts have been performed to create soft sensors, under different low-dimensional empirical and physical modeling frameworks directly in the process computers, see e.g. Karlström and Eriksson (2014a,b). These approaches have also enabled the development of new measurement systems and methods for information extraction that often set limits on the digital storage resources. In many cases this is handled by decimating (filtering and downsampling) the signals already in the distributed control algorithms. Since downsampling reduces the sampling rate, some important information can be lost for future analysis if the Nyquist-Shannon sampling theorem is not maintained, see Nyquist (1928), Shannon (1949) and Jerri (1977).

Even when the decimation is performed properly, the process computers still provide a huge amount of information. This information contains both oversampled and undersampled variables in many cases, i.e. oversampled process data provided at a higher sampling rate compared with undersampled variables such as pulp property analysis from laboratory tests. The latter can be seen as data residing in a much lower dimensional space. Undersampled data sets are most often obtained with non-equidistant sampling intervals while the process variables from the distributed controllers are equidistantly oversampled. This problem was originally overlooked in several traditional control concepts, as pointed out by Johansson et al. (1980), Dahlqvist and Ferrari (1981), Oksum (1983), Honkasalo et al. (1989), Karlström and Koebe (1993) and Hill et al. (1993), However, Hill et al. (1979) already proposed control strategies in the 1970s that used on-line pulp quality and consistency measurements as a complement to specific energy (or motor load) control to cope with the problem.

A variety of approaches have been used for comparing the impact of different process variables on the refining conditions. For example, Strand and Hartler (1985), Strand et al. (1993), Strand (1996), Schwartz et al. (1996), Lidén (2003), Lama et al. (2006) and Harrison et al. (2007) used multivariable statistical methods when analyzing effects on pulp quality variables.

Some 40 years ago, relationships between refining zone temperature measurements and pulp quality variables were already being studied by Atack (1975) and Stationwala et al. (1979), but no evidence for correlations of this kind was found. Several initiatives to equip TMP refiners with temperature sensors for process monitoring and control have recently been proposed. The measurements delivered by such sensors have been found to contribute substantially to an increased understanding of refining processes, see Johansson (2001), Mosbye et al. (2001), Berg et al. (2003), Karlström et al. (2005) Johansson and Richardson (2005), Sikter et al. (2007), Karlström et al. (2008) and Ferritsius et al. (2014).

To handle dynamic information that is valuable for the analysis of the pulp property development, Karlström and Eriksson $(2014 \mathrm{a}, \mathrm{b})$ concluded that the viscosity and force distribution inside the refining zone were most important. 


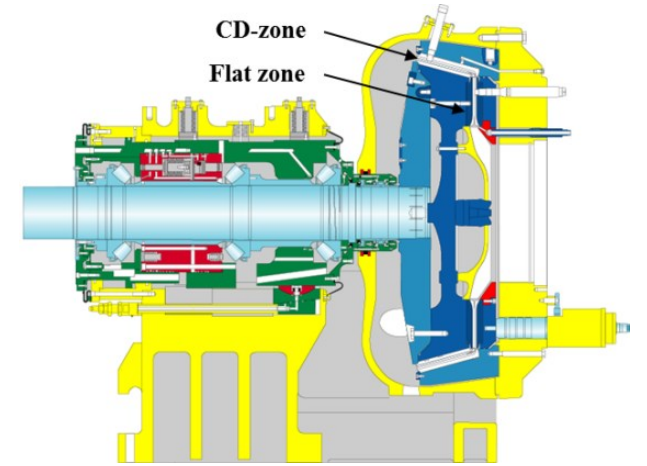

Fig 1 - A schematic drawing of a CD-refiner. The vertical flat zone $(F Z)$ is directly linked to the conical zone (CD) via an expanding point.

This paper gives an example of how to combine oversampled variables from the process computer and undersampled variables from laboratory measurements, using a piece-wise linear approach to get a common time frame for further analysis. The tensile index, mean fiber length (MFL), Somerville shives content (from here on abbreviated 'Somerville') can be seen as typical external states. These were analyzed using internal states in terms of the fiber residence time and the estimated consistency vector inside the refining zone.

This paper is the first in a series of three articles where the intention is to link laboratory pulp measurements with process data to study different dynamic phenomena.

\section{Material and Methods}

In the present study, process data sets were analyzed from a full-scale production line (CD82-refiner) at the Stora Enso Kvarnsveden mill in Sweden.

The CD82-refiner ${ }^{1}$, see Fig 1, consists of two serially linked refining zones called the flat zone (FZ) and the conical zone (CD). In both zones, temperature sensor arrays have been mounted to measure the entire temperature profiles. To link these internal states to the external states, traditionally monitored process variables such as production rate, dilution water flows, plate gaps and motor load were measured as well.

The mean fiber length (MFL) was measured on-line using a pulp sampling device. However, this measurement was not useful except as a model reference due to a slow sampling rate as well as varying delays and time constants in the latency. Instead, samples were taken in the blow-line, with a careful sampling procedure using several partial samples for a well-documented sampling time. Specialized equipment made it possible to take about 30 partial samples during a three-minute period.

To get a reliable set of data for the pulp property analysis, 63 pulp samples from five test periods $\{$ TEST $1, \ldots$, TEST5 $\}$ were obtained from the CD82refiner.

The pulp samples can be seen as a set of undersampled variables, which must be expanded into an oversampled data set including 350000 samples of traditional process variables to get a common time frame.

\footnotetext{
1 The raw material is Scandinavian spruce and the CD-refiner is equipped with a $25 \mathrm{MW}$ motor running at $1800 \mathrm{rpm}$.
}

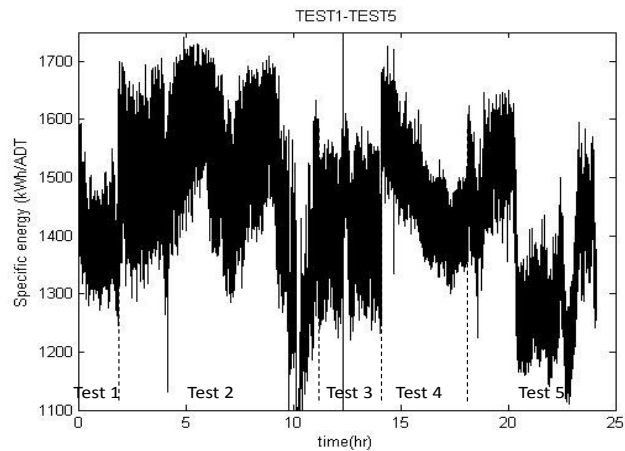

Fig 2 - Specific energy for each test series TEST1 $\rightarrow$ TEST5. The time on the x-axis is not continuous and illustrates only the intervals for each test series.

Table 1 - Changes in the manipulated variables for the test series studied. Mean values and the step/ramped change in the plate gaps, dilution water feed rates and production.

\begin{tabular}{|c|c|c|c|c|c|}
\hline $\begin{array}{l}\text { Manipulated } \\
\text { variable }\end{array}$ & TEST1 & TEST2 & TEST3 & TEST4 & TEST5 \\
\hline $\begin{array}{l}\text { Plate gap } F Z \text {, } \\
\text { mm }\end{array}$ & 1.5 & $\begin{array}{l}1.3 \\
0.9 \\
1.3\end{array}$ & 1.2 & 1.5 & 0.9 \\
\hline $\begin{array}{l}\text { Plate gap CD, } \\
\mathrm{mm}\end{array}$ & 0.9 & 0.8 & $\begin{array}{l}0.63 \\
0.56 \\
0.63\end{array}$ & 1.1 & 0.7 \\
\hline $\begin{array}{l}\text { Dilution water } \\
\mathrm{FZ}, \mathrm{l} / \mathrm{s}\end{array}$ & 3.3 & $\begin{array}{l}3.4 \\
3.1 \\
3.4\end{array}$ & 3.8 & 3.4 & $\begin{array}{l}3.3 \\
3.55 \\
3.3\end{array}$ \\
\hline $\begin{array}{l}\text { Dilution water } \\
\text { CD, I/s } \\
\text { Production, } \\
\text { t/h }\end{array}$ & $\begin{array}{l}4.8 \\
13.5\end{array}$ & 15.3 & 15.8 & $\begin{array}{l}12.4 \\
14.1 \\
15.8\end{array}$ & $\begin{array}{l}4.7 \\
4.4 \\
14.5\end{array}$ \\
\hline
\end{tabular}

TEST1 was primarily performed to analyze the variance in process conditions and pulp quality without changing the set points for the process variables. The set point for the plate gaps, the dilution waters and the production rate were then systematically changed in TEST2-TEST5 according to Table 1. The present paper focuses on the tensile index, MFL and Somerville variations based on measurements from laboratory tests.

The test series were spread non-equidistantly over the period Nov. 2012 to Jan. 2013 and covered a large dynamic operating window. In this paper, however, each test series will be visualized on a common $\mathrm{x}$-axis and it is understood that each period is non-equidistantly sampled, see example in

Fig 2 where the specific energy is shown for each test series. According to Karlström and Eriksson (2014a,b), the consistency profile in the refining zone and the blowline can be estimated if measurements of the temperature profiles and plate gaps are available together with a physical model. The model was called "the extended entropy model" and is given in the appendix. Using this model, the fiber residence time may be extracted as well.

Miles and May (1990) found that fiber residence time plays an important role in describing the fiber-to-bar impact in the refining zone. Originally, the fiber residence time was an average of the total residence time in the refining zone and was not very well documented 
from a modeling perspective. Later, Härkönen et al. (1999) showed by using radioactive tracers that the fiber residence time varies depending on where the detector was positioned along the refining segment radius; the authors concluded that the fiber pad is spread out tangentially and radially, simultaneously.

In the literature, the residence time is exclusively related to the pulp, without considering the effect of chip, pulp and fiber. Karlström and Eriksson (2014a,b) commented on this point, but the theory was only vaguely linked to the residence time via the chip, pulp and fiber viscosity variations. It is therefore assumed in this paper that the residence time refers to the fiber residence time, i.e. the average transport time of fibers from the inner part of the refining zone to the outside rim of the refining segments, as outlined by Karlström and Hill (2014a) ${ }^{2}$.

$$
\tau=\int_{r_{\text {in }}}^{r_{\text {out }}} \frac{1}{v_{1}(r)} d r=\int_{r_{\text {it }}}^{r_{\text {out }}} \frac{\rho_{1}(r) A_{1}}{m_{1}(r)} d r
$$

The density, $\rho_{l}$, mass flow, $m_{1}$, the cross-area available for the pulp, $A_{1}$, and thereby the pulp velocity, $v_{l}$, can be derived from the extended entropy model. The residence time, $\tau$, between arbitrary positions in the refining zone $\left\{r_{i n}, r_{\text {out }}\right\}$ can therefore be estimated as well.

Karlström and Isaksson (2009) introduced the concepts of internal and external states. The internal states referred to physical variables obtained from refining zone measurements or estimates from physical models while external states referred to process inputs and outputs such as dilution water and chip feed rates, motor load and pulp properties.

As the test series cover a long period and a large dynamic operating window, non-linearities such as process limitations and wear of the refining segments must also be considered. A linear regression approach applied to the complete data set will therefore most often fail even though reliable process conditions are used. This is best illustrated in Fig 3, where the estimated MFL from a $1^{\text {st }}$ order polynomial fit is given using the specific energy input as an independent variable. Some improvements can be obtained by increasing the polynomial degree, but this is still not good enough as the residual variance from the fitted model is too low $\left(1^{\text {st }}\right.$ order $\mathrm{R}^{2} \approx 0.3,2^{\text {nd }}$ order $\mathrm{R}^{2} \approx 0.44,3^{\text {rd }}$ order $\left.\mathrm{R}^{2} \approx 0.45\right)^{3}$.

To cope with non-linearities in the process, another approach is to introduce piece-wise linear functions, see Hamann and Chen (1994). These are functions composed of straight-line sections, i.e. domains decomposed into pieces on which the function is linear ${ }^{4}$. Using this type of

2 The residence time estimation according to $E q 1$ is a simplification that does not properly account for the known fiber recirculation before the maximum temperature. However, the impact of fiber recirculation on the pulp property development has not been proved, although studied by Stationwala et al. (1987) and Härkönen et al. (1999).

3 If performing the same estimation for tensile index and Somerville the following residual variances are obtained: ( 1 st order $\mathrm{R}^{2} \approx 0.25,2^{\text {nd }}$ order $R^{2} \approx 0.25$ ) and ( 1 st order $R^{2} \approx 0.65,2^{\text {nd }}$ order $R^{2} \approx 0.66$ ) respectively.

4 The only restriction is that the non-linearities must be able to be approximated as piece-wise linear functions. To some extent, this approach has much in common with the non-parametric multiplicative

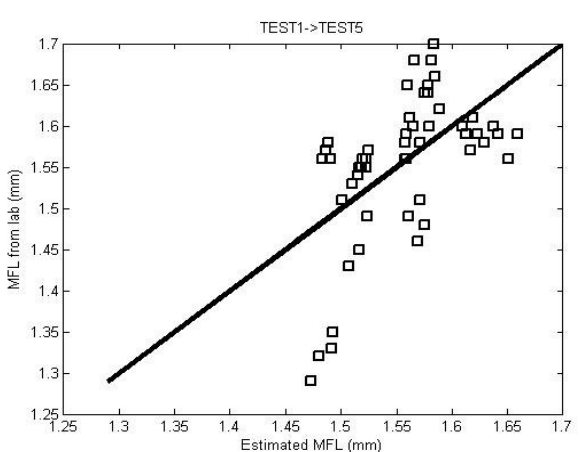

Fig 3 - Estimated and measured MFL from the 63 pulp samples obtained during five test periods. The straight line corresponds to the optimal fit when using specific energy as an independent variable.

approach, the internal variables play an important role, as hidden physical phenomena that are impossible to measure can be used to improve the pulp property estimation.

During the test series, the pulp sampling $t_{S}$ was obtained during an interval of one minute and three minutes for TEST1 while in all other test series (TEST2 $\rightarrow$ TEST5) the interval was set to three minutes to get a representative averaged sample from the blow-line.

As the process data had a sampling frequency of $4 \mathrm{~Hz}$ for each pulp sampling interval of three minutes, the number of process measurements is considerable and knowing the time for each batch of pulp samples, $y_{m}$, including the time interval, it is possible to specify a function $\hat{y}_{m}(t)$ according to

$y_{m_{i}}=0 \rightarrow \hat{y}_{m}(t)=\hat{f}\left(x_{m}\right) ; m=\{$ Test $1, \ldots .$, Test 5$\}$

$y_{m_{i}} \neq 0 \rightarrow \hat{y}_{m_{i}} \approx \bar{y}_{m_{i}}$; The mean $\bar{y}_{m_{i}}$ is formed by the $n$ samples. where $i$ different pulp properties can be considered and $x_{m}$ can be any internal state coupled to the pulp property $y_{m}$ studied. $x_{m}$ is assumed to be a scalar, but in those cases where $\mathbf{x}_{\boldsymbol{m}}$ is a vector, the non-linearities in each test series can be modeled by multiple piece-wise linear equations which must not have any discontinuities. In other words, a non-linear function $f\left(\mathbf{x}_{\mathbf{m}}\right)$ is modeled as a collection of linear functions of the form

$\hat{f}_{j}\left(\mathbf{x}_{\mathbf{m}}\right)=$

$\theta_{j 1} x_{m 1}+\theta_{j 2} x_{m 2}+\cdots+\theta_{j k} x_{m k}+b_{j} \quad \forall j=1,2, \ldots, q$.

where $\left\{\theta_{j 1}, \ldots, \theta_{j k}\right\}$ represents the parameter vector. The number of linear regions into which the non-linear function is broken up is represented by $q$. If the function is non-linear in one independent variable, the total number of regions is simply the number of line segments used to represent the function.

When process data from different periods are used, it is often impossible to describe the boundaries. This is true also in this study and means that a set of discrete functions, describing the non-linearities for each test series, with no continuity between the line segments, will be used.

regression approach, see Corder and Foreman (2009), with local linear estimators for which the traditional process variance and standard deviations are useful tools in the analysis. 
Several internal variables can be used as independent variables in the piece-wise linear functions, and the variable selected in this work is naturally the fiber residence time, which indirectly links to the major refining conditions and to the refining segment pattern used.

It is assumed here that the pulp properties can be related to the residence time by using piece-wise linear functions, and this assumption can be questioned as we have not shown how the residence time varies with the pulp properties. Moreover, the residence time according to Karlström and Hill (2014a) can be seen as an approximation in this context as long as the "absolute" residence time is not verified. Nevertheless, due to the possibility of splitting total residence time into parts representing sections on the refining segments, it should be possible to analyze the residence time and its impact on the pulp properties in a wider perspective.

The sum of the residence time in the flat zone $\left(\tau_{F Z}\right)$ and in the conical zone $\left(\tau_{C D}\right)$ is primarily used as an independent variable, and the estimated pulp properties are derived using the 2 -norm of the residuals $r_{i}$, i.e. the differences between the observations, $y_{i}$ and the model, $r_{i}=y_{i}-\hat{y}_{i} ; i=1, \ldots m$, are used when selecting the linear function using the criterion $\min \left\|r_{i}\right\|_{2}$.

Some of the pulp properties measured might require an extension where a weight function is introduced in the norm ${ }^{5}$; sometimes both refining zones must be considered simultaneously to get a good estimate. This opens the way for multiple piece-wise linear functions as independent variables when predicting the pulp properties, which will be commented further below.

\section{Results and Discussion}

During the five different test periods, the consistency in the blow-line was carefully manually measured, which made it possible to cross-check the result from the extended entropy model ${ }^{6}$. As seen in Fig 4, the extended entropy model seemed to provide a good estimation of the consistency in the periphery of the $\mathrm{CD}$ zone segments, even though the estimated consistency was slightly higher for some of the sampling points compared with the manually measured consistency in the blow-line.

The residence time in different parts of the refining zone is also linked to the local consistency, and it is possible to show how much a change in inlet consistency

5 If many outliers are expected in the data population, an extended procedure using weighted least squares and minimizing the 2-norm

$\|r\|_{2}=\sqrt{\sum_{i=1}^{m} w_{i} r_{i}^{2}}$

can be used. The term $w_{i}$ represents different weights that can be introduced if some observations are more accurate than others. Different refining segment designs can introduce spatial dependencies that must also be considered. For instance, the outer part of the CDzone might require a weight function.

6 The inlet consistencies in the chips to the refining zone were calibrated for each test series to give the best fit to the outlet consistencies. Inlet consistencies used were TEST1-50\%, TEST2$56 \%$, TEST3-53\%, TEST4-53\% and TEST5-51\%. (or change in dilution water feed rate) influences the total residence time at different production rates, see Karlström and Hill (2014a,b). The relationship is nonlinear and dependent on the actual operating conditions; Karlström and Eriksson (2014a,b) showed that the segment design, disc velocity and radial steam pressure gradients have a huge impact on the final residence time in different parts of the refining zone.

Fig 5 shows the residence time estimations for all five tests. The residence time fluctuated considerably in the two refining zones depending on the actual process conditions ${ }^{7}$. This effect is even more pronounced in Fig 6 , which plots the ratio between residence time in the flat zone and the total residence time. The deviation in specific energy input, shown in

Fig 2 does not correlate with this residence time ratio, in either the flat or the conical zones.

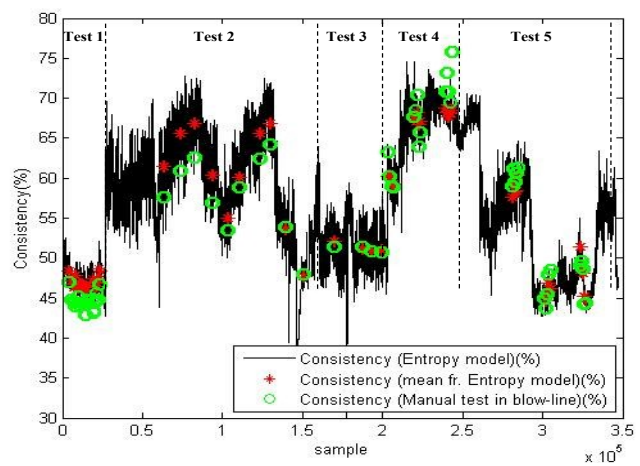

Fig 4 - Estimated periphery consistency in the CD zone using the extended entropy model (black) and its mean values (red) when taking pulp samples (green) from the blow-line ${ }^{8}$. The time on the $\mathrm{x}$-axis is not continuous and illustrates only the intervals for each test series.

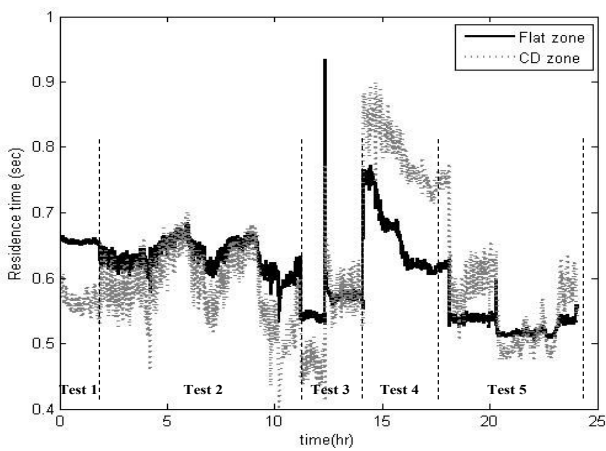

Fig 5 - Estimated residence time in the flat zone (FZ) and the conical zone (CD) for the five test series studied. The time on the $\mathrm{x}$-axis is not continuous and illustrates only the intervals for each test series.

\footnotetext{
${ }^{7}$ Even though the length of the $C D$ segments is about $75 \mathrm{~mm}$ longer compared with the FZ segments, the residence time there can be shorter. This means that the fiber pad will be treated differently depending on which refining zone is being studied.

8 Note, in Fig. 3 , the samples are used on the $x$-axis only to show how the undersampled variables can be interlaced into a large population (sampling rate $0.25 \mathrm{sec}$.) of estimated process conditions. The rest of the paper uses a time basis of one hour.
} 


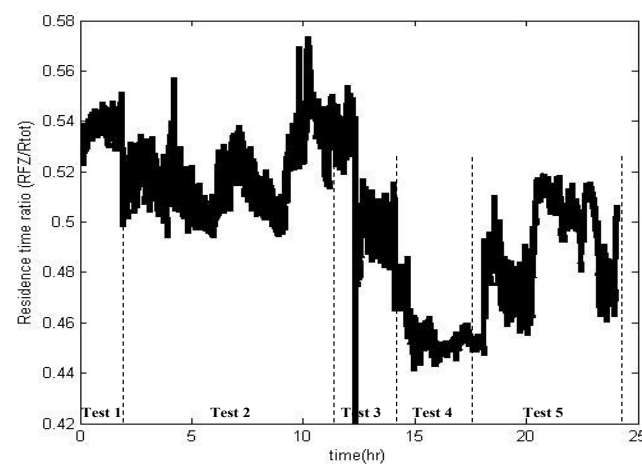

Fig 6 - Estimated ratio between the residence time in the flat zone (FZ) and the total residence time versus time intervals for all five tests. The time on the $x$-axis is not continuous and illustrates only the intervals for each test series.

\section{Influence of residence time}

In the present study, the model structures are not in focus. Instead, the results from the models will be presented in plots together with the laboratory measurements provided. A more extensive discussion about model structures will be given later in this series of papers.

Mean fiber length: The procedure using piece-wise linear functions described above can be visualized by plotting MFL versus the residence time in both the FZ and CD. In Fig 7, which represents the laboratory data from TEST 2, the 2-norm for the conical zone is about one third of the 2-norm in the flat zone. From a statistical perspective, this leads to a selection of $\tau_{C D}$ as an independent variable in the piece-wise linear function when estimating MFL for TEST 2. The residence time differed rather a lot between the test series as seen in Fig 8 , where MFL is plotted versus the total residence time for TEST2 and TEST5. This makes it difficult to prespecify which refining zone is the most important, and how other internal variables affect the estimates.

Even though other internal variables can be used as well, the more rudimentary piece-wise linear functions seem to give useful approximations, at least for short time test intervals, as can been seen in Fig 7. Note that the online measured MFL follows the estimation, although it is biased, and delayed presumably due to the latency chest ${ }^{9}$, see Fig 9.

From a dynamic perspective, the expanded estimation clearly followed the on-line measured MFL. This strengthens the situation, since using the total residence time as the independent variable resulted in a relatively good model with an $\mathrm{R}^{2}$ of 0.91 , i.e. the model explains $91 \%$ of the variance in MFL. Using the specific energy input as the independent variable resulted in a smaller $\mathrm{R}^{2}$ of 0.81 .

An extension of the modeling procedure by using a multiple regression approach, i.e. the coefficients in $E q 2$ are determined using Gauss's least squares regression, using the local residence time in FZ and CD together with the inlet consistency and the outlet consistency in the

${ }^{9}$ In this paper, the on-line device was not calibrated to give values comparable to the laboratory data.

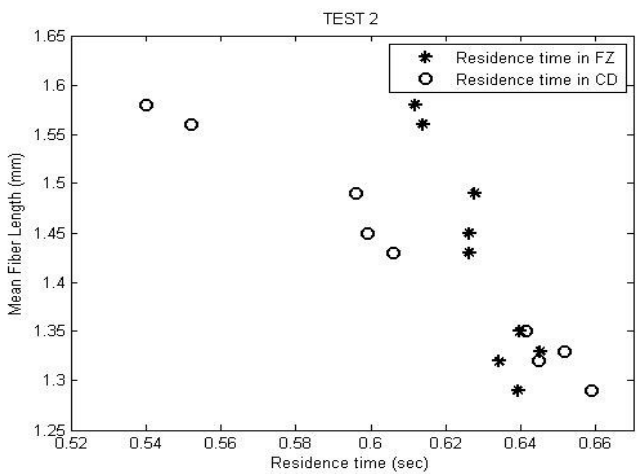

Fig 7 - Measured MFL in TEST2 versus the estimated residence time in flat zone (FZ) and conical zone $(C D)$.

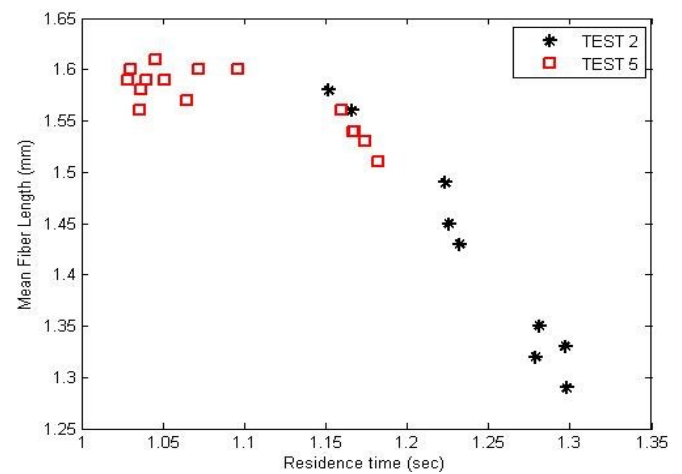

Fig 8 - Measured MFL versus total residence time in TEST2 and TEST5.

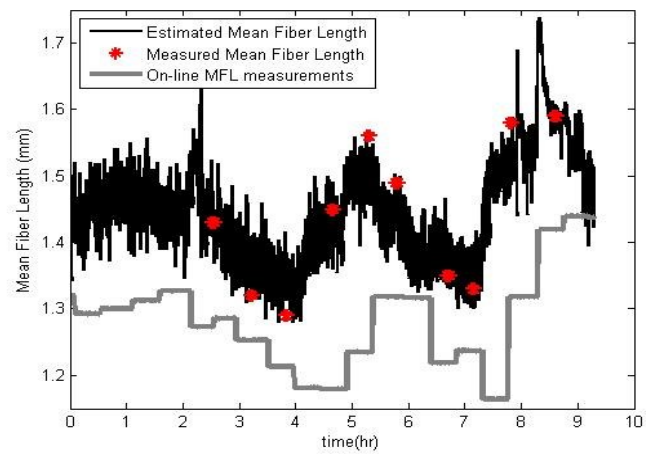

Fig 9 - Laboratory measured MFL (red dots), on-line measured MFL (grey) and estimated MFL using the total residence time as an independent variable based on data from TEST2.

blow-line, will result in a slightly better estimate where $\mathrm{R}^{2}=0.94$.

Even though a penalty for the number of terms in the model (in total four independent variables) is included, the model extension is motivated from a statistical perspective ${ }^{10}$. Note that the models derived for each test series are not comparable, due to process non-linearities. Therefore, it is not possible to make a traditional validation. This statement will be discussed later in this series of papers.

10 That is, $R^{2}$ is adjusted based on the residual degrees of freedom. The residual degree of freedom is defined as the number of response values minus the number of fitted coefficients estimated from the response values. An increased set of parameters reduces the $R^{2}$. Later, this measure will be called Adjusted R². 


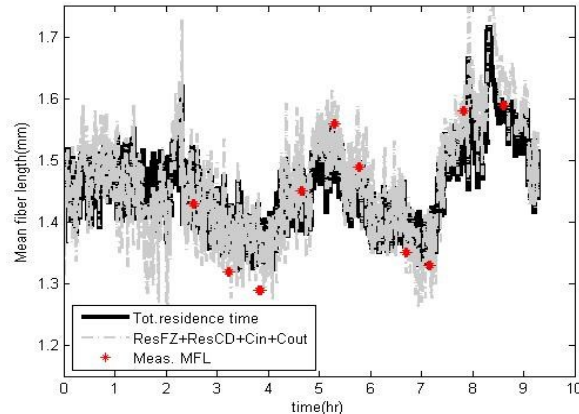

Fig 10 - Laboratory measured MFL (red dots) and estimated MFL using the total residence time as an independent variable (black) and MFL estimated from residence time and consistency in FZ and CD (grey). The data set is based on TEST2.

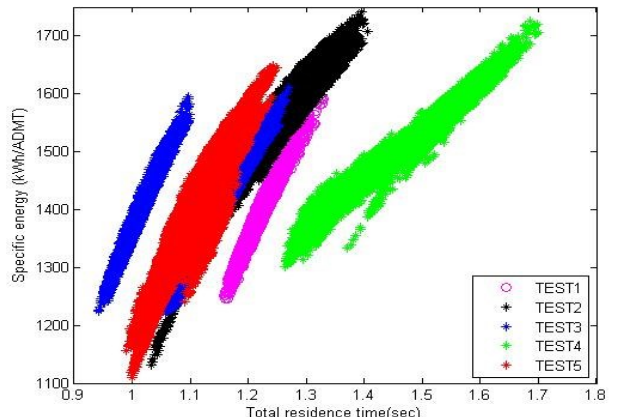

Fig 11 - Specific energy input versus total residence time obtained from the extended entropy model.

However, a common drawback when using several independent variables, estimated from process variables with much noise, is that the model sensitivity often increases ${ }^{11}$ which conceals the improvement. This can be handled by pre-filtering the process variables or the independent variables before applying the regression analysis.

The improvement in $\mathrm{R}^{2}$ is best illustrated by comparing the model with the total residence time as the only independent variable, and the model with residence time in each refining zone together with inlet and outlet consistencies as independent variables. Using the same filtering parameters for both cases, the improvements are clearly visible; see Fig 10. The dynamic response in the estimated MFL seems to be acceptable. The results suggest that MFL drops when residence time increases ${ }^{12}$. This can be strengthened to some extent by studying Fig 11, where the total residence time trends are monotonically increased with increased specific energy input for all test series, see Karlström and Hill (2014a).

Tensile index: In this paper, all pulps have been tested twice for sheet properties, i.e., for each sample, the number of strips for tensile index was doubled based on an average of 40 strips.

\footnotetext{
11 The variance in the estimate is doubled primarily owing to the consistency fluctuations.

12 In this study, the assumption is certainly valid for TEST2 while, for the other test series, the best fit is somewhat weaker. The magnitudes of variations of the MFL are most pronounced in TEST2, which can be a result of the operation close to the limit of the operating window. This statement will be discussed in later articles in this series of papers.
}
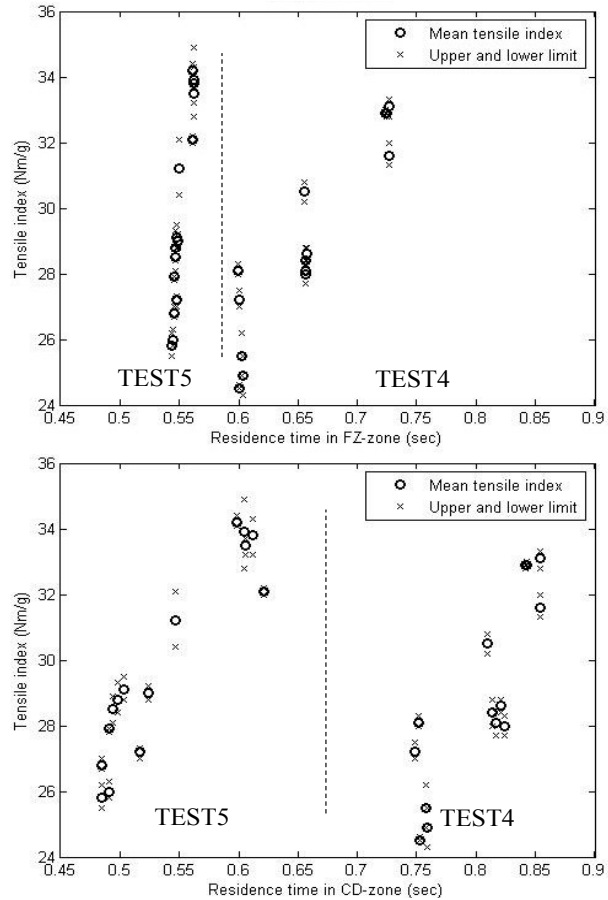

Fig 12 - Measured tensile index versus residence time in the flat zone and the conical zone, respectively. Upper figure refers to the flat zone and lower figure to the CD zone.

As can be seen in Fig 12, two distinct sets of data referring to TEST4 and TEST5 were obtained when the tensile index was plotted versus the residence time. The production rate changes in TEST4, according to Table 1, affected the residence time in both the flat and conical zones simultaneously.

In TEST5, where the dilution water flow rates to each zone were changed, the residence time was only slightly affected by the changes in the flat zone. Applying the selection procedure given in $E q$ 2, a set of piece-wise linear functions was obtained. It is clear from the $\mathrm{R}^{2}$ that the fit was improved when using both local residence times and consistencies in the FZ and CD, as compared with a situation in which the total residence time was used, see Table 2. It is interesting to see that increased complexity, for instance by introducing the production rate as an independent variable, does not improve the fit. This may be explained by the fact that the local residence times and consistencies were estimated from internal variables, such as temperature, and external variables, such as production rate and motor load.

In Fig 13, the tensile index is estimated for the complete set of tests using the same approach as in Fig 7. The dynamics seemed to be sensitive to process changes in a similar way as to MFL. If this is true, the process conditions inside the refining zone must be kept as stable as possible to get more uniform pulp properties.

The tensile index is a complex pulp property from another perspective and comprises more information about the process conditions than MFL. The tensile index normally increases with residence time. However, for specific process conditions, this assumption must be reconsidered, as there may be situations where the tensile index decreases with residence time. This will be further explored below and in future papers in this series. 
Table 2 - Goodness of fit in terms of adjusted $R^{2}$.

\begin{tabular}{lccc}
\hline $\begin{array}{l}\text { Estimation of tensile index } \\
\text { Independent variables }\end{array}$ & $\begin{array}{c}\text { No of } \\
\text { variables }\end{array}$ & $\begin{array}{c}\text { Adj. } \mathbf{R}^{\mathbf{2}} \\
\text { TEST4 }\end{array}$ & $\begin{array}{c}\text { Adj. } \mathbf{R}^{\mathbf{2}} \\
\text { TEST5 }\end{array}$ \\
\hline $\begin{array}{l}\text { Total residence time } \\
\begin{array}{l}\text { Residence time and Consistency } \\
\text { in FZ\&CD }\end{array}\end{array}$ & 1 & 0.81 & 0.87 \\
$\begin{array}{l}\text { Residence time and Consistency } \\
\text { in FZ\&CD and Prod }\end{array}$ & 5 & 0.91 & 0.96 \\
\hline
\end{tabular}

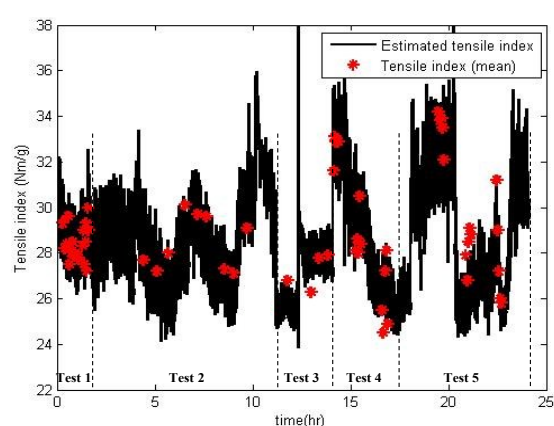

Fig 13 - Estimated and measured tensile index for the periods (TEST1-TEST5) studied.

\section{Influence of Different Refiner Consistencies on Pulp Properties}

As shown in Fig 14, the consistency exiting the flat zone during TEST2 was too high as compared with common practice. This means that the steam generation was approaching the limit where the forward flowing steam is negligible. When the steam flow in that position is dramatically reduced, the traditional three phase system is locally affected, which means that the risk for fiber cutting is imminent (Strand 2014, Liukkonen et al. 2014).

The temperature profile information from TEST2 provides valuable insight into the phenomena in the flat zone. This can be seen in Fig 15, in which a photo of the sensor array has been inserted. The plot shows that the temperature of the outer sensor (Position 8), near the periphery of the flat zone, does not increase when the consistency out from the flat zone is more than $80 \%$. As a consequence, not enough steam can be generated in that position. The inner temperature sensors in Fig 15, represented by Positions 6 and 7, were still responding when the plate gap and dilution water feed rate were reduced in TEST2, which prompted us to analyze the distributed consistency in greater detail.

It is important to mention that it is not possible to estimate the consistency variations in the refining zones if the temperature profile measurements are not available.

Clearly, however, the relationship between the pulp properties and the consistencies and other refining zone variables is complex, since the manipulated variables were considerably different between the test series, see Table 1. The complexity is best illustrated by studying plots of pulp properties versus consistency.

Mean fiber length: As seen in Fig 16, MFL was found to decrease with increasing consistency exiting the flat zone. This statement was true for all test series, even though the decrease was small for TEST3 and TEST4. It is interesting to observe that the slopes in TEST2 and TEST5 are similar when compared with the other test series, even though TEST5 was obtained two months after TEST2.

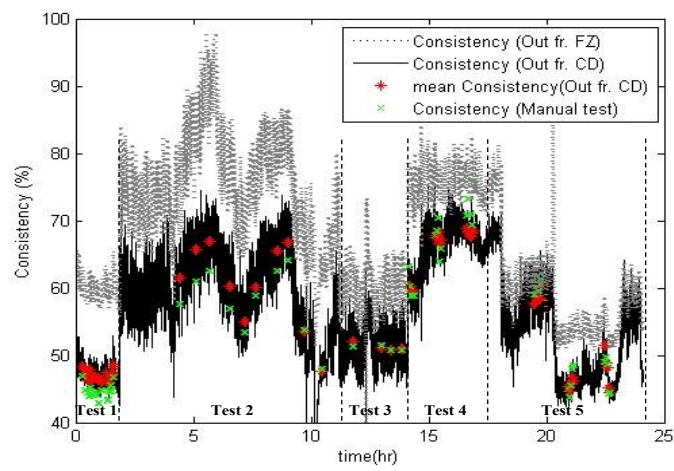

Fig 14 - Estimated consistencies exiting the flat and conical zones together with the manual test of the consistency in the blow-line.

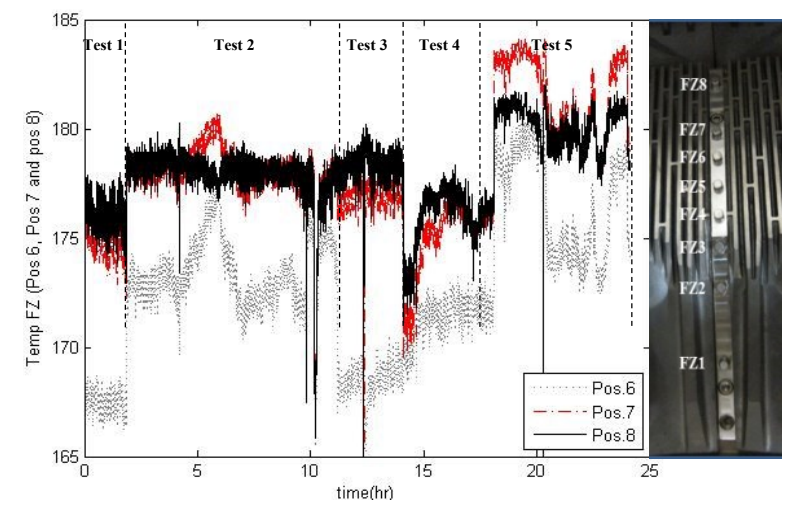

Fig 15 - Left: Temperature measurements in the outer part of the flat zone. Right: Positions 6-8 are indicated as FZ6-FZ8.

Tensile Index: If the tensile index development is dependent on the consistency vector in the refining zone, in our case the flat zone, it is natural to plot the estimated tensile index versus the consistency in the outer part of the flat zone, similar to the case for MFL. As indicated in Fig 14, only TEST2 seemed to reach typical consistencies over $80 \%$ in the outer part of the flat zone while the consistencies in TEST1, TEST3, TEST4 and TEST5 were less than $80 \%{ }^{13}$, see Fig 17 . The tensile index is strongly dependent on the how the fiber pad is developed at different consistencies in the refining zones, and the data obtained from TEST2 showed a totally different slope when running at the edge of the refining capacity, see

Fig 18. The consistency was higher than normal in the flat zone, which indicates that a maximum for the tensile index is reached if the consistency is increased beyond a certain point.

Somerville: The Somerville shives values for each test series tended to follow the slope of the MFL versus consistency in the flat zone, as seen in Fig 19. TEST3 showed a different slope and may not be representative. Note that the estimated Somerville is clustered differently as compared with MFL.

13 The flat zone periphery consistency has been studied by Backlund (2004) but in general it is difficult to measure or estimate it on-line. Note, for TEST4 it is also higher than expected. 


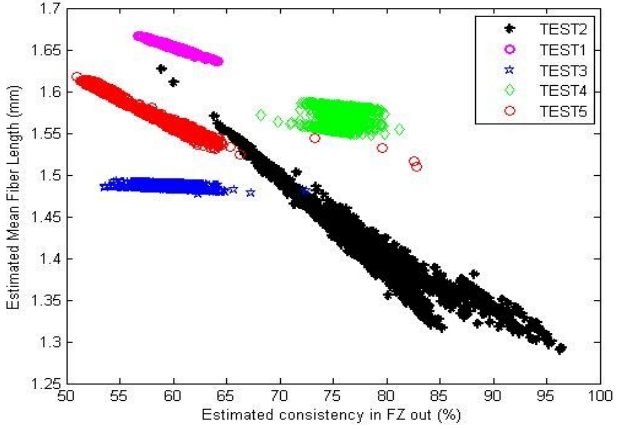

Fig 16 - Estimated MFL versus estimated consistency in the flat zone for all tests performed.

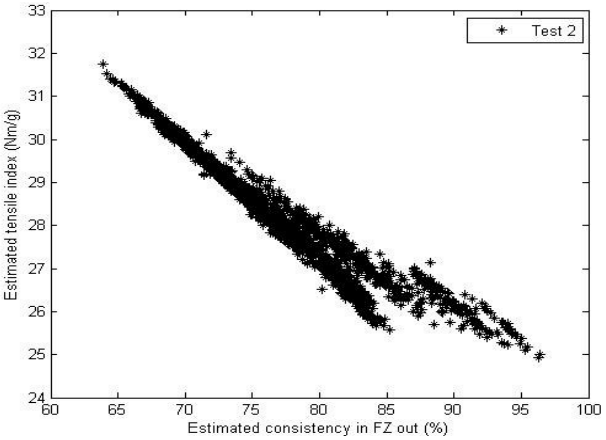

Fig 18 - Estimated tensile index versus estimated consistency in the flat zone for TEST2.

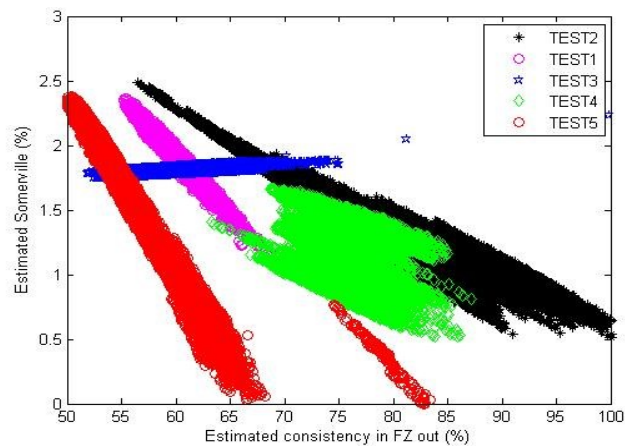

Fig 19 - Estimated Somerville shives versus estimated consistency in the flat zone for all tests performed.

\section{Conclusions}

The main purpose of this study was to investigate the possibility of interlacing undersampled pulp properties (63 laboratory tests) and oversampled TMP process data (>350 000 samples), from a commercial CD82-refiner, using piece-wise linear functions as approximations.

It was shown that the residence time and consistency in each refining zone are vital when estimating the mean fiber length, tensile index and Somerville shives. Furthermore, the residence time can be split in order to cope with variations in both the flat zone and the conical zone. The consistency in the flat zone and the blow-line consistency also have an important role in obtaining a good polynomial fit. Goodness of fit $\left(\mathrm{R}^{2}\right)$ was improved when using both local residence times and consistencies in FZ and CD compared with a situation where the total residence time is used alone as an independent variable.

By expanding the laboratory data and matching them with estimates from piece-wise linear functions, it was

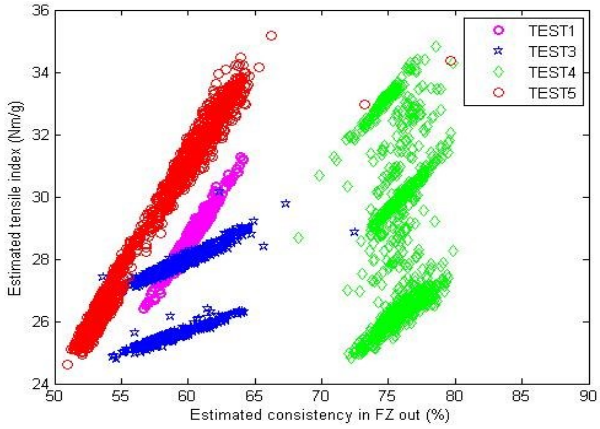

Fig 17 - Estimated tensile index versus estimated consistency in the flat zone.

possible to analyze them in a new way as compared with traditional methods. The different tests covered a number of process conditions, requiring that the data sets be handled judiciously. The drawback of the methodology using piece-wise linear functions is that several models are needed to span the entire process operating window. This is a consequence of the process non-linearities and, therefore, it is natural to propose complementary methods to analyze the differences in the process operating window dynamically. This will be studied in more detail in later papers in this series.

\section{Acknowledgements}

The authors gratefully acknowledge funding by the Swedish Energy Agency, StoraEnso, SCA and Holmen Paper. Special thanks to Stora Enso Kvarnsveden mill for performing trials and providing excellent laboratory and process data used in this study.

\section{Literature}

Atack D., Stationwala M.I. (1975): On the measurement of temperature and pressure in the refining zone of an open discharge refiner, International Mechanical Pulping Conference, San Francisco, California, USA, p. 135-142.

Backlund, H.-O. (2004): "Measurement of shear force, temperature profiles and fibre development in mill-scale TMP refiners", Licentiate Thesis, Mid Sweden University, Sundsvall, Sweden.

Berg D., Karlström A. and Gustavsson M. (2003): Deterministic consistency estimation in refining processes, International mechanical pulping conference, Quebec, Canada, p. 361-66.

Corder, G.W. \& Foreman, D.I. (2009): Nonparametric Statistics for Non-Statisticians: A Step-by-Step Approach, Wiley. ISBN 978-0-470-45461-9.

Dahlqvist G. and Ferrari B. (1981): Mill operating experience with a TMP refiner control system based on a true disc clearance measurement, International Mechanical Pulping Conference, Oslo, Norway, Session III, no. 6.

Eriksson, K., Karlström, A. (2009): Refining Zone Temperature Control: A good choice for Pulp Quality Control? International Mechanical Pulping Conference in Sundsvall, Sweden.

Ferritsius, R., Hill, J., Ferritsius, O., Karlström, A. (2014): On Energy Efficiency in Chip Refining. International Mechanical Pulping Conference 2014 in Helsinki, Finland. 
Hamann, B. and Chen, J.L. (1994): Data point selection for piecewise linear curve approximation, Computer Aided Geometric Design, Elsevier, Volume 11, Issue 3, Pages 289301.

Harrison R.P., Roche A.A. and Stuart P. (2007): Impact of refining line interruptions and reject refiner operation on pulp and paper variability, Tappi J., 6(12), p. 3-9.

Hill J., Westin H. and Bergström R. (1979): Monitoring pulp quality for process control, International Mechanical Pulping Conference, Toronto, Canada, p. 111-125.

Hill J., Saarinen K. and Stenros R. (1993): On the control of chip refining systems, Pulp and paper Canada, 94(6), p. 43-47.

Honkasalo J.V., Polkkynen E.E. and Vainio J.A. (1989) Development of control systems in mechanical pulping (GW, TMP) at Rauma, International Mechanical Pulping Conference, Helsinki, Finland, p. 376-389.

Härkönen, E., Huusari, E. amd Ravila, P. (1999): Residence time of fiber in a single disc refiner, Int. Mech. Pulping Conf., pp. 77.

Jerri, A.J. (1977):The Shannon Sampling Teorem - Its Various Extensions and Applications: A Tutorial Review, Proc IEEE,Vol 65, No 1 Nov 1977.

Johansson B.-L., Karlsson H. and Jung E. (1980): Experiences with computer control, based on optical sensors for pulp quality, of a two-stage TMP-plant, 1980 Process Control Conference, Halifax, Nova Scotia, p. 145-152.

Johansson 0. (2001): Controlling high consistency refining conditions through refining zone temperature optimisation, $6^{\text {th }}$ Pira International refining conference, Toronto, Canada.

Johansson 0. and Richardson J. (2005): The effect of refining zone temperature on refining efficiency and pulp quality, International Mechanical Pulping Conference, Oslo, Norway.

Karlström A. and Koebe M. (1993): Modelling of wood chip refining processes, Nordic Pulp and Paper Research Journal 8(4), p. 384-388.

Karlström A., Berg D. and Eriksson K. (2005): Developments in soft sensors for measurement of refining parameters, Scientific and technical advances in refining and mechanical pulping, Barcelona, Spain, Paper 5.

Karlström A., Eriksson K., Sikter D. and Gustavsson M. (2008): Refining models for control purposes, Nordic Pulp and Paper Research Journal 23(1), p. 129-138.

Karlström A. and Isaksson A. (2009): Multi-rate optimal control of TMP refining processes, International Mechanical Pulping Conference, Sundsvall, Sweden.

Karlström, A. and Eriksson, K. (2014a): Fiber energy efficiency Part I: Extended entropy model. Nord. Pulp Paper Res. J 29(2), Pages 322-331.

Karlström, A. and Eriksson, K. (2014b): Fiber energy efficiency Part II: Forces acting on the refiner bars. Nord. Pulp Paper Res. J 29(2), 332.

Karlström, A. and Hill J. (2014a): Refiner Optimization and Control Part I: Fiber residence time and major dynamic fluctuations in TMP refining processes. Nord. Pulp Paper Res. J. 29(4), 635-652.
Karlström, A. and Hill J. (2014b): Refiner Optimization and Control Part II: Test procedures for describing dynamics in TMP refining processes. Nord. Pulp Paper Res. J. 29(4), 653-662.

Lama I., Perrier M. and Stuart P. (2006): An empirical model for predicting motor load changes due to plate wear in TMPrefiners, Nordic Pulp and Paper Research Journal 21(4), p. 527-533.

Lidén J. (2003): Quality control of single stage double disc chip refining, Licentiate thesis, Mid Sweden University, Sundsvall, Sweden.

Liukkonen, S., Nurminen, I., Fredrikson, A., Salminen, L., Sirviö, J. (2014): Development of pulp properties within refiner plate gap in energy efficient and cutting processes. International Mechanical Pulping Conference, Helsinki, Finland.

Miles, K. B. and May, W. D. (1990): The Flow of Pulp in Chip Refiners, J. Pulp Paper Sci. 16(2), 63.

Mosbye K., Kure K.-A., Fuglem G. and Johansson O. (2001): Use of refining zone temperature measurements for refiner control, International Mechanical Pulping Conference, Helsinki, Finland, p. 481-488

Nyquist H. (1928): Certain topics in telegraph transmission theory, AIEE Trans., Vol 47, pp 617-644.

Oksum J. (1983): New technology in the Skogn mechanical pulp mill, International Mechanical Puling Conference, Washington DC, USA, p. 143-153.

Schwartz H.M., Chang G. and Liu Y. (1996): A method for predicting and controlling TMP pulp properties, IEEE International Conference on Control Applications, Dearborn, USA.

Shannon C.E. (1949):Communication in the presence of noise, Proc. IRE, Vol.37, pp 10-21,Jan 1947.

Sikter D., Karlström A., Engstrand P. and Czmaidalka J. (2007) Using the refining zone temperature profile for quality control, International Mechanical Pulping Conference, Minneapolis, Minnesota, USA.

Stationwala M.I., Atack D., Wood J.R., Wild D.J. and Karnis A. (1979): The effect of control variables on refining zone conditions and pulp properties, International Mechanical Pulping Conference, Toronto, Ontario, Canada, p. 93-109.

Stationwala M.I., Atack D., and Karnis A. (1987): Effect of refiner plate design on the development of pulp properties, International Mechanical Pulping Conference, Vancouver, B.C, Canada, p. 1-10.

Strand, B.C,. Hartler, N. (1985): Modelling and optimization of full scale chip refining IMPC(1985) pp. 46.

Strand, B.C., Mokvist, A, Falk, B., Jackson, M. (1993): The effect on production rate on specific energy consumption in high consistency chip refining IMPC 1993, pp. 143.

Strand, B.C. (1996): Model based control of high consistency refining, TAPPI Journal 79(10), pp.140-146.

Strand, B.C., Grace, B. (2014): Implementation of advanced supervisory control within a TMP refiner quality control system, International Mechanical Pulping Conference, Helsinki, Finland.

Manuscript received May 29, 2015 Accepted September 18, 2015 


\section{Appendix}

This appendix summarizes the entropy and enthalpy balances. As an example, the energy balances in the flat zone according to Karlström and Eriksson (2014a) are given, see Fig 20. Note that this block is one of several blocks that are set by the number of temperature sensors used in the sensor array.

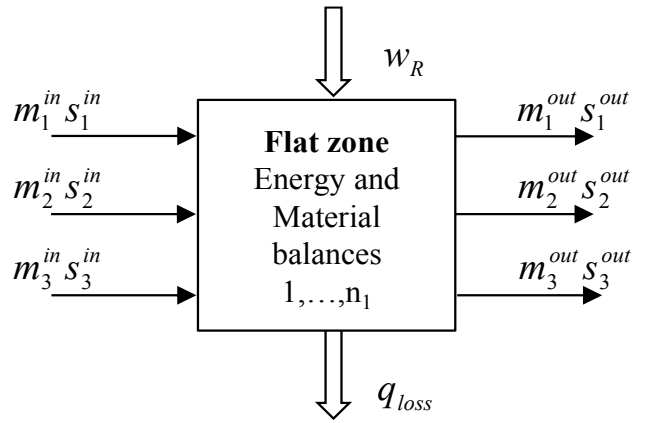

Fig 20 - Energy balance for a hypothetical refining zone.

$d S(r)=\frac{\delta(r)}{T(r)} \Delta(r) 2 \pi r d r$

where

$\delta(r)=\mu\left(\frac{r \omega}{\Delta(r)}\right)^{2}=\frac{w_{R}(r)}{\Delta(r)}$

We also know that

$$
\begin{gathered}
d S(r)=m_{1} c_{p} \ln \left(\frac{T(r+d r)}{T(r)}\right)+ \\
\sum_{j=2}^{3}\left(m_{j}(r+d r) s_{j}(r+d r)-m_{j}(r) s_{j}(r)\right) \\
w_{t h}(r) 2 \pi r d r=m_{1} c_{p}(T(r+d r)-T(r))+ \\
\sum_{j=2}^{3}\left(m_{j}(r+d r) h_{j}(r+d r)-m_{j}(r) h_{j}(r)\right)
\end{gathered}
$$

which gives

$$
\begin{aligned}
& \frac{w_{R}(r)}{T(r)} 2 \pi r d r=m_{1} c_{p} \ln \left(\frac{T(r+d r)}{T(r)}\right)+ \\
& \qquad \sum_{j=2}^{3}\left(m_{j}(r+d r) s_{j}(r+d r)-m_{j}(r) s_{j}(r)\right) \\
& m_{2}(r)+m_{3}(r)=m_{2}(r+d r)+m_{3}(r+d r) \\
& w_{R} \neq 0 \text { and } q_{\text {loss }} \approx 0 \\
& m_{1}^{\text {in }}=m_{1}^{\text {out }}=m_{1}, m_{2}^{\text {in }} \text { and } m_{3}^{\text {in }} \text { are known } \Rightarrow \\
& m_{2}^{\text {in }}+m_{3}^{\text {in }}=m_{2}^{\text {out }}+m_{3}^{\text {out }} \Rightarrow m_{2}^{\text {out }}=m_{2}^{\text {in }}+m_{3}^{\text {in }}-m_{3}^{\text {out }} \\
& d r=r_{\text {out }}-r_{\text {in }}
\end{aligned}
$$

Find $m_{3}^{\text {out }}, w_{t h_{\text {in }}}$ and $w_{d e f_{i n}}$

$$
\begin{aligned}
& X=\frac{w_{R_{\text {in }}}}{T_{\text {in }}} 2 \pi r_{\text {in }} d r-m_{1} c_{p} \ln \left(\frac{T_{\text {out }}}{T_{\text {in }}}\right)-m_{2}^{\text {in }}\left(s_{2}^{\text {out }}-s_{2}^{\text {in }}\right)- \\
& \quad m_{3}^{\text {in }}\left(s_{2}^{\text {out }}-s_{3}^{\text {in }}\right) \\
& Y=m_{1} c_{p}\left(T_{\text {out }}-T_{\text {in }}\right)+m_{2}^{\text {out }} h_{2}^{\text {out }}-m_{2}^{\text {in }} h_{2}^{\text {in }}+m_{3}^{\text {out }} h_{3}^{\text {out }}- \\
& \quad m_{3}^{\text {in }} h_{3}^{\text {in }} \\
& m_{3}^{\text {out }}=X /\left(s_{3}^{\text {out }}-s_{2}^{\text {out }}\right) \\
& w_{\text {thin }}=Y /(2 \pi r d r) \\
& w_{\text {def }_{\text {in }}}=w_{R_{\text {in }}}-w_{\text {th }}
\end{aligned}
$$

Table 3 - Latin symbols

\begin{tabular}{ll}
\hline Symbol & Description \\
\hline$c_{p}$ & Heat capacity \\
$d S$ & Entropy generation \\
$h_{i}$ & Specific enthalpy of component $i$ \\
$m_{i}$ & Material flow of component $i$ \\
$q_{l o s s}$ & Energy losses per unit area \\
$R$ & Radial coordinate \\
$s_{i}$ & Specific entropy of component $i$ \\
$S$ & Total entropy \\
$T$ & Temperature \\
$w_{t h}$ & Thermodynamic work per unit area \\
$w_{d e f}$ & Refining work per unit area \\
$w_{R}$ & Estimated total work per unit area
\end{tabular}

Table 4 - Greek symbols

\begin{tabular}{ll}
\hline Symbol & Description \\
\hline$\delta$ & Viscous dissipation \\
$\mu$ & Dynamic viscosity \\
$\omega$ & Angular speed of the refiner disc \\
$\Delta$ & Plate gap at radius $r$
\end{tabular}

Table 5 - Indices

\begin{tabular}{ll}
\hline Sub- and superscript & Interpretation \\
\hline 1 & Wood/pulp phase \\
2 & Water phase \\
3 & Steam phase \\
in & Refiner inlet \\
out & Refiner outlet \\
\hline
\end{tabular}

